\section{Making and Using Aqueous Mounting Media, or Why Buy a Premixed Medium when it's Cheap and Easy to make your own? John Kiernan, University of Western Ontario}

Instructions are given as follows for making five aqueous mounting media: glycerol jelly, buffered glycerol with anti-fade, fructose syrup, Apathy's gum-syrup, and a polyvinylpyrrolidone medium whose composition can be varied to suit the user's needs. For each, hints are given on usage and the preservation of the coverslipped preparations. Media made in the laboratory are cheaper than commercial aqueous mountants, and do not contain any secret ingredients.

Pretty well everything sold for use in a laboratory is outrageously expensive, and the price gets even higher when things are marketed for one particular purpose rather than for general use. Water-based mounting media are an example. They consist almost entirely of such cheap chemicals as gum. gelatin and sugar, yet an inspection of recent catalogs shows that the cheapest aqueous mountant costs more than a dollar per milliliter. That works out at 10 cents or more for each slide, on top of the cost of the glass and whatever staining reagents were used.

It is a simple matter to make your own aqueous mounting media, at almost no cost. The only pieces of equipment you need are a few small beakers or flasks, a source of heat, and either a lot of elbow grease or a magnetic stirrer. As well as monetary savings. there is another good reason to mix your own: you know what you've got. A commercial mounting medium may be excellent for what you want to do, or it may not. If the formulation is a trade secret, there's no way of knowing until after you've bought the stuff.

Here are instructions for making a few aqueous mounting media. They are not all the same, because there isn't a single medium that can be used for every purpose. With the exception of some of the antibacterial additives, no dangerous chemicals are used in these mixtures, so you won't need to wear a space-suit when mixing them. Once made, aqueous mounting media should be stored in small ( 10 to $30 \mathrm{~mL}$ ) screw-capped bottles or vials made of transparent glass or plastic. The cap can get cemented in place, but is usually removable by soaking in warm water. If the medium becomes cloudy or shows other signs of bacterial or fungal growth, throw it out and open another bottle.

Aqueous media are much used for fluorescence microscopy. and some of these mixtures contain substances that retard the fading of fluorochromes. Some of the media set or dry to become fairly solid: others are always liquid. With all of them it is advisable to seal the edges of the coverslip if you want a permanent preparation. Use nail varnish or some similar material that does not mix with water. Any resinous mounting medium, diluted with an equal volume of toluene or xylene, can be used in the same way as nail varnish.

\section{Glycerol jelly:}

This traditional mounting medium is the most difficult one to use. If you can make a decent preparation in glycerol jelly, you'll be able to use any other aqueous mountant with your eyes shut.

Gelatin powder: $10 \mathrm{~g}$

Water: $60 \mathrm{~mL}$

Dissolve by warming and add:

Glycerol: $70 \mathrm{~mL}$

Add either one drop of saturated aqueous solution of phenol ("liquid phenol") or $15 \mathrm{mg}$ of sodium merthiolate as an antibacterial agent.

This can be kept for a few weeks at $4^{\circ} \mathrm{C}$. Discard when it becomes turbid or moldy.

Glycerol jelly must be must be warmed to about $40^{\circ} \mathrm{C}$ to melt the gel before using. It commonly needs to be freed of air bubbles, too. This can be done by warming the bottle (with cap loosened) in a vacuum-embedding chamber.

After coverslipping with glycerol jelly, leave the slide on a warm $\left(40^{\circ}\right.$ to $45^{\circ}$ C), flat surface for about 30 minutes, to let the medium soak into the section, and then remove it to a cool place to set It is quite difficult to make a bubble-free preparation with this medium, and the bubbles can be tiny and numerous. Check with a microscope while the slide is still warm. If it's no good, remove the coverslip by soaking in warm water, and try again.

This medium has a low refractive index (1.42). Because of this, many unstained structures remain visible, which may be an advantage or a disadvantage, depending on what you expect to see in the finished preparation.

\section{Buflered glycerol with anti-fade:}

Buffered red glycerol is used mainly for fluorescent immunohistochemical preparations. The high $\mathrm{pH}$ provides for optimally efficient fluorescence of the commonly used labels. The added p-phenylenediamine (PPD) (Platt \& Michael, 1983) or n-propyl gallate (Longin et al., 1993; Battaglia et al., 1994), retards fading.

Buffer:

Either $0.1 \mathrm{M}$ Phosphate buffer $(\mathrm{pH} 7.4): 10 \mathrm{~mL}$

or $0.1 \mathrm{M}$ TRIS buffer ( $\mathrm{pH} 9.0)$ : $10 \mathrm{~mL}$

Anti-fading agent:

Either $p$-phenylenediamine hydrochloride: $100 \mathrm{mg}$

or $n$-propyl gallate: $500 \mathrm{mg}$

Glycerol: $90 \mathrm{~mL}$

Keeps for at least 3 months, probably much longer, in darkness (which protects the anti-fade agent) at $-20^{\circ} \mathrm{C}$. The working bottle is kept at $4^{\circ} \mathrm{C}$, for a week or two.

This does not solidify, but the coverslip can be held in position by applying a little nail varnish to lts edges.

\section{Fructose syrup:}

This medium is sticky enough to hold a coverslip in position. It is the easiest aqueous medium to use, but too acid (pH about 4.5) for preserving basic dyes. Probably it could be made up in a neutral buffer; I have never tried that myself. Fructose syrup is useful when you know you will be removing the coverslip to do something else to the section.

Fructose (=levulose): $15 \mathrm{~g}$

Water: $5 \mathrm{~mL}$

Put together in a securely capped bottle and leave in an oven (about $60^{\circ} \mathrm{C}$ ) for 1 to 2 days, until all the sugar has dissolved to form a clear syrup.

Keeps for several months on the shelf at room temperature.

With long storage this medium will dry out and crystallize if the edges of the coverslip are not sealed.

\section{Apathy's gum-syrup:}

This is another traditional mountant: troublesome to make, but easy to use. The thymol is to retard the growth of microorganisms. Another disinfectant (see under glycerol jelly) could be used instead.

Gum arabic (=gum acacia): $50 \mathrm{~g}$

Sucrose: $50 \mathrm{~g}$

Water: $50 \mathrm{~mL}$

Thymol: one small crystal

Dissolve the ingredients, with frequent stirring and occasional heating on a water bath. If big lumps form, the gum may take several days to dissolve. The final volume should be approximately $100 \mathrm{~mL}$. Keeps for a few months at room temperature. Discard if it becomes infected or if the sugar crystallizes. Sanderson (1994) recommends fructose (levulose) instead of sucrose, presumably because it is less likely to crystallize.

The refractive index of Apathy's medium (about 1.5) is higher than that of glycerol jelly or fructose, and it provides preparations that are almost as transparent as those made with a resinous mounting medium. 
Polyvinylpyrrolidone (PVP) medium:

This is my favorite aqueous mounting medium. The composition can be varied according to individual needs (Kiernan, 1990). For immunofluorescence, make it up in a buffer and add an anti-fading agent.

Polyvinylpyrrolidone (M.W. 10,000): $25 \mathrm{~g}$

Water (or a $0.1 \mathrm{M}$ phosphate or TRIS buffer, pH 7.4 or 9): $25 \mathrm{~mL}$

Dissolve the PVP by leaving for several hours on a magnetic stirrer. Then add:

Glycerol:1.0 mL

Thymol: one small crystal

Polyvinyl alcohol can be used instead of PVP, and either pphenylenediamine hydrochloride ( $10 \mathrm{mg}$ per $100 \mathrm{~mL}$ ) or $n$-propyl gallate $(600 \mathrm{mg}$ per $100 \mathrm{~mL}$ ) may be added, if desired, to inhibit fading of fluorescence (Valnes \& Brandtzaeg, 1985).

Bottles of PVP medium usually keep for 2 to 3 years at room temperature. Keep it in a dark place if an anti-fading agent has been added. Discard if it looks infected or becomes too viscous.

This mounting medium is more runny than glycerol jelly or Apathy's. It is very easy to apply, and not prone to bubbles. The refractive index is 1.46 (Pearse, 1968), but increases as the water evaporates at the edges of the coverslip until unstained structures are barely visible. If you want a high degree of transparency, wait several days before sealing the edges of the coverslip.

Battaglia, M., Pozzi, D., Grimaldi, S. and Parasassi, T. 1994. Hoechst 33258 staining for detecting mycoplasma contamination in cell cultures: a method for reducing fluorescence photobleaching. Biotech. Histochem. 69:152-156.

Kiernan, J. A. 1990. Histological and Histochemical Methods. Theory and Practice. 2nd ed. Oxford: Pergamon Press.

Longin, A., Souchier, C., Ffrench, M. and Bryon, P. A. 1993. Comparison of anti-fading agents used in fluorescence microscopy: image analysis and laser confocal microscopy study. J. Histochem. Cytochem, 41:1833-1840.
Pearse, A. G. E. 1968. Histochemistry, Theoretical and Applied, 3rd edn. Vol.1 Edinburgh: Churchill-Livingstone.

Platt, J. L. and Michael, A. F. 1983. Retardation of fading and enhancement of intensity of immunofluorescence by $\rho$-phenylenediamine. J. Histochem. Cytochem. 31 840-842.

Sanderson, J. B.1994. Biological Microtechnique (Royal Microscopical Society Handbook No.28) Oxford: BIOS Scientific Publishers.

Valnes, K. and Brantzaeg, P. 1985. Retardation of immunofluorescence fading during microscopy. J. Histochem. Cytochem. 33: 755-761.

\section{No-More MSA Reprints!!!}

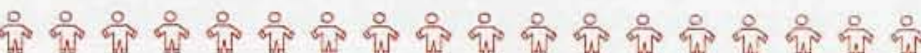

As you may recall, in the past we have occasion ally reprinted papers from the MSA/EMSA Conferences which we felt would be of interest to our readers. We will not be allowed to do this in the future.

I wish to make it VERY clear that this is NOT a decision by the Microscopy Society of America. And, just as we have in the past, we intend to support MSA in every way we can.

It seems that MSA has retained a "professional" publisher, Springer-Veriag, to handle their journal and proceedings. As one could say that we "compete" for advertising income, they have advised "...from a practical standpoint, it makes no sense for us to grant such permission."

Kindly note our restraint in not making further comment!

\section{-Don Grimes, Editor}

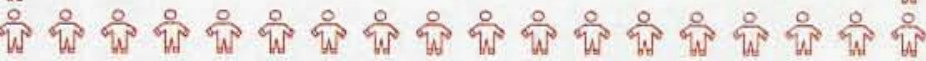

\section{STOP HASSLING WITH
MULTIPLE SERVICE CONIRAGTS}

START by putting all of your instruments under one contract with MAS

(regardless of make or model). Our expert EM SERVICE GROUP has the knowledge and skill to keep your instrumentation working at its best.

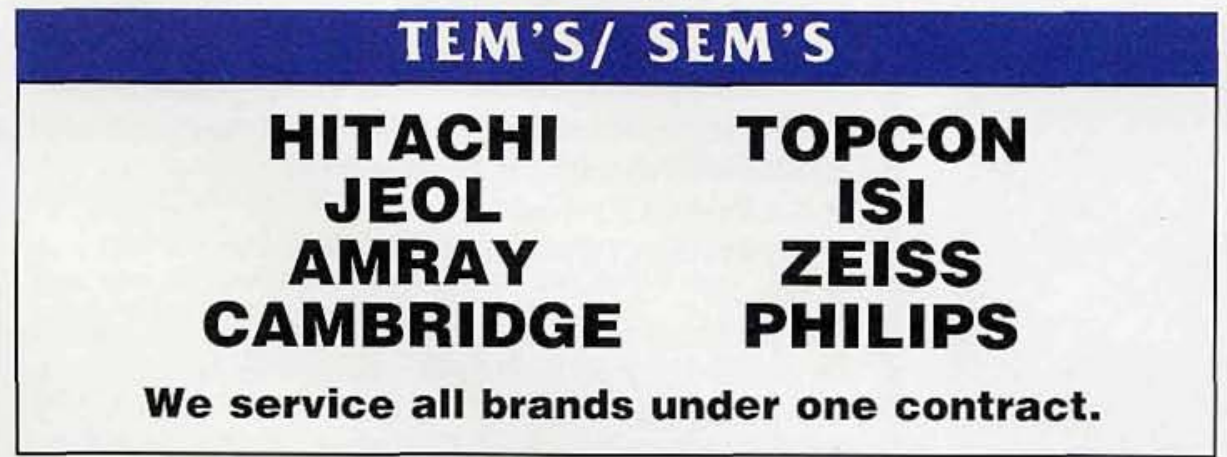

PREP EQUIPMENT

ULTRAMICROTOMES DUPONT \& RMC

VACUUM COATERS SPUTTER COATERS MECHANICAL PUMPS TURBO PUMPS PLASMA ASHERS

\begin{tabular}{|c|}
\hline WE SERVICE \\
\hline COMPUTER CONTROLLERS \\
BEAM BLANKERS \\
WATER CHILLERS \\
\hline
\end{tabular}

Contracts and On-Demand Emergency Service at Reasonable Rates from Factory Trained Specialists. 1-800-421-8451

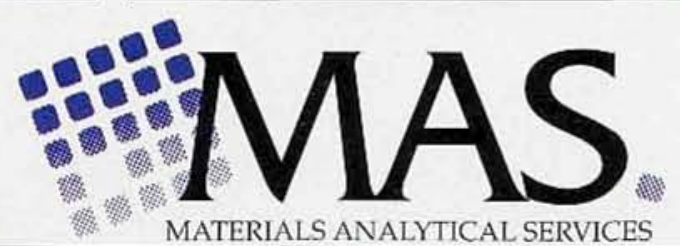

3597 Parkway Lane • Suite 250 • Norcross, Georgia 30092 • 770-448-3200 • FAX 770-368-8256 or 616 Hutton Street • Suite 101 • Raleigh, North Carolina 27606 • 919-829-7041 • FAX 919-829-5518 ADVANCED ANALYTICAL PRODUCTS AND SERVICES 